\title{
Implementation of Standardized Instructions for Insulin at Hospital Discharge
}

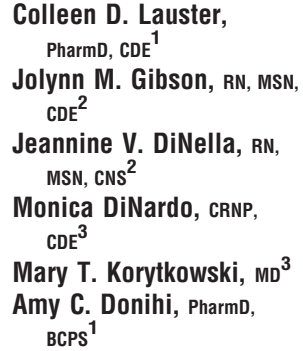

Colleen D. Lauster, PharmD, $\operatorname{CDE}^{1}$

Jolynn M. Gibson, RN, MSN, $\mathrm{CDE}^{2}$

Jeannine V. DiNella, RN, MSN, CNS ${ }^{2}$

Monica DiNardo, CRNP, $\operatorname{CDE}^{3}$

Mary T. Korytkowski, mo ${ }^{3}$

Amy C. Donihi, PharmD, BCPS $^{1}$

\author{
${ }^{1}$ Department of Pharmacy and Therapeutics, School of Pharmacy, University of Pittsburgh, \\ Pittsburgh, Pennsylvania. \\ ${ }^{2}$ Department of Nursing, University of Pittsburgh Medical Center, Pittsburgh, Pennsylvania. \\ ${ }^{3}$ Division of Endocrinology, School of Medicine, University of Pittsburgh, Pittsburgh, Pennsylvania.
}

Insulin is designated a high-alert medication because of its potential to result in harm if it is used incorrectly. ${ }^{1}$ Despite this, changes in insulin regimens made in the inpatient setting are often poorly communicated to either the patient or his primary care physician at the time of discharge. ${ }^{2}$ Poor communication of medication instructions at the time of hospital discharge has been linked to medication errors and adverse drug events. ${ }^{3}$

We conducted a quality improvement project to improve and standardize the communication of insulin instructions to patients (and/or their caregivers) at hospital discharge. Specifically, we developed and implemented a standardized discharge instructions for insulin (DIFI) form and compared the comprehensiveness of insulin instructions and diabetesrelated readmissions before and after the introduction of the form.

\section{Methods}

A multidisciplinary team ${ }^{4}$ created the DIFI form. Page 1 (Figure 1) includes sections for entering all insulin types and doses and the frequency of glucose monitoring and a space for specific diabetes instructions. Page 2 provides general information on symptom recognition and management of hyperglycemia and hypoglycemia. Page 3 is a blank glucose log.

We retrospectively reviewed the records of patients discharged to home on insulin from a general medicine unit during the 3-month period before availability of the DIFI form and during the 3-month period afterward. Approval for this project was obtained from the hospital's quality improvement review committee. The percentages of orders with specific instructions for the timing and dosing of basal, prandial, and correction insulin and home glucose monitoring were calculated. The number of patients readmitted within 2 weeks of discharge for a diabetes-related problem was also determined. Fisher's exact tests were used to compare demographics and indicators in the preimplementation and postimplementation groups.

\section{Results}

Chart review was performed for 67 patients with insulin orders at discharge prior to the DIFI form and for 27 patients after implementation. There were no group differences in gender (female gender: $63 \%$ pre-DIFI vs. $63 \%$ postDIFI, $P=0.49)$, previous history of diabetes $(98.5 \%$ vs. 92.6\%, $P=0.20$ ), diabetes-related admitting diagnosis (20.1\% vs. $37 \%, P=0.12$ ), or insulin use prior to admission (95.5\% vs. $85.2 \%, P=0.10$ ).

More orders written with the DIFI form contained specific instructions for timing and dosing of basal (67\% vs. $100 \%, P=0.0003$ ), prandial (51\% vs. $100 \%, P=0.0008)$, and correction insulin ( $14 \%$ vs. $95 \%, P<0.0001)$ and for glucose monitoring ( $17.9 \%$ vs. $88.9 \%, P<0.0001)$. There were 4 diabetes-related readmissions in the preimplementation group and none in the postimplementation group $(P=$ not significant).

\section{Discussion}

It is important that patients receive clear directions at the time of hospital discharge to ensure a safe transition of care from the inpatient setting to the outpatient setting. This is particularly true for insulin regimens, which frequently consist of at least 2 different types of insulin and often include instructions for modifying doses on the basis of home glucose readings. The day of hospital discharge is not conducive to recall of verbal communication concerning complicated medication regimens. ${ }^{5}$ Additionally, our hospital's standard discharge form was not a satisfactory tool for providing detailed directions. We found that the DIFI form prompted a more consistent provision of specific instructions for insulin therapy and glucose monitoring in comparison with previous practice. 
UPMC

DISCHARGE INSTRUCTIONS FOR INSULIN

MPRINT PATENT DENTFCATION HERE

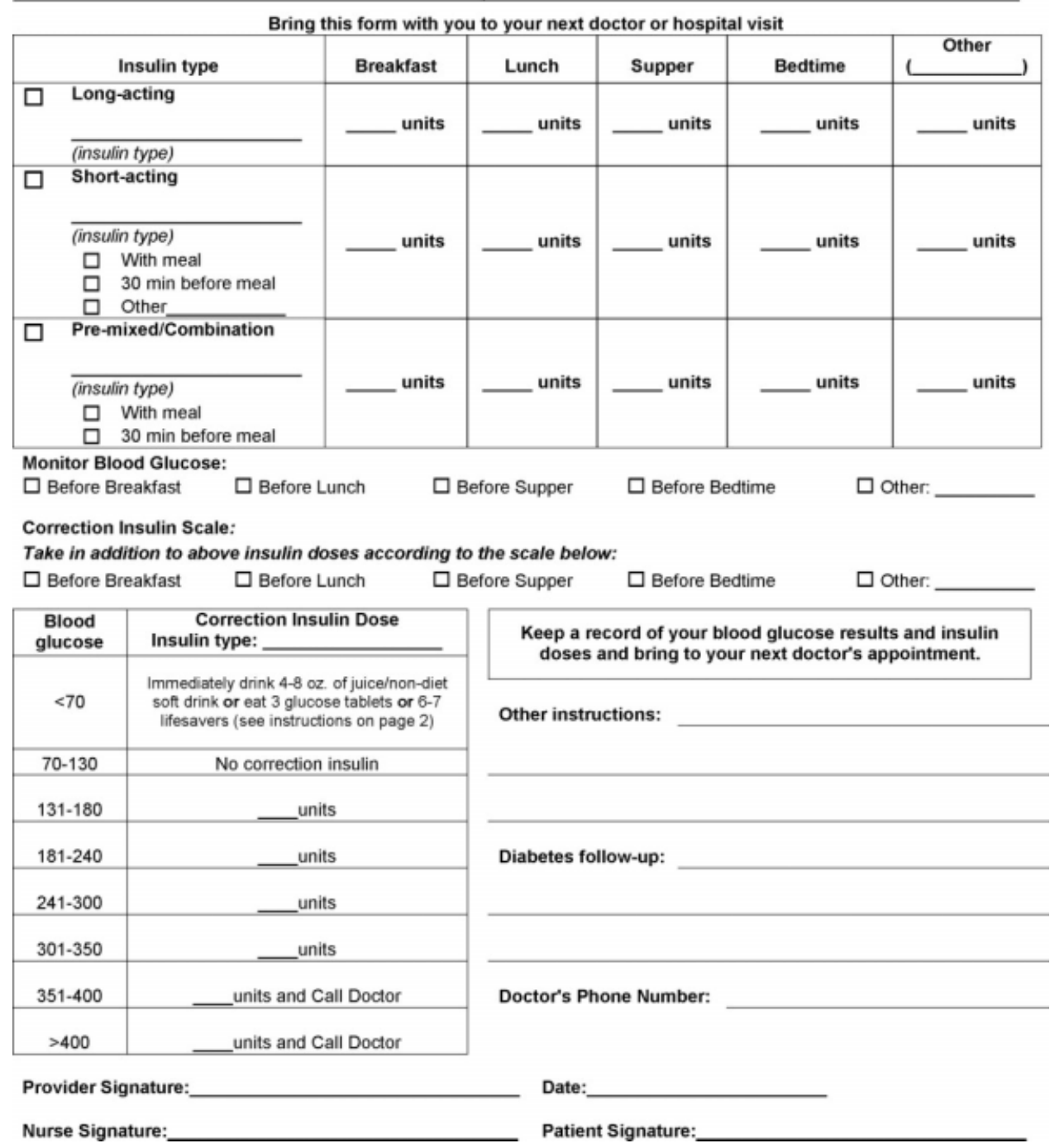

FIGURE 1. Page 1 of the discharge instructions for insulin form.

\section{References}

1. Institute for Safe Medication Practices. ISMP's list of high-alert medications. Available at: http://www.ismp.org/tools/highalertmedications.pdf. Accessed December 2008.

2. Kripalani S, Jackson AT, Schnipper JL, Coleman EA. Promoting effective transitions of care at hospital discharge: a review of key issues for hospitalists. J Hosp Med. 2007;2:314-323.
3. Forster AJ, Murff HJ, Peterson JF, Gandhi TK, Bates DW. The incidence and severity of adverse events affecting patients after discharge from the hospital. Ann Intern Med. 2003;138:161-167.

4. Korytkowski M, DiNardo M, Donihi AC, Bigi L, DeVita M. Evolution of a diabetes inpatient safety committee. Endocr Pract. 2006;12(suppl 3):91-99.

5. Donihi AC, Yang E, Mark SM, Sirio CA, Weber RJ. Scheduling of pharmacist-provided medication education for hospitalized patients. Hosp Pharm. 2008;43:121-126. 